International Journal of Engineering \& Technology, $7(1.1)(2018) 642-650$
International Journal of Engineering \& Technology
Website: $w$ ww.sciencepubco.com/index.php/IJET
Research paper

\title{
Flower image classification with basket of features and multi layered artificial neural networks
}

\author{
Syed Inthiyaz $^{1 *}$, B.T.P.Madhav ${ }^{2}$, Ch. Raghava Prasad ${ }^{3}$ \\ ${ }_{1,2,3}$ Department of Electronics and Commutation Engineering \\ K.L.E.F. Deemed to be University, Green Fields, Vaddeswaram, Guntur, A.P., INDIA-522 502 \\ *Corresponding author E-mail: syedinthiyaz@kluniversity.in
}

\begin{abstract}
Artificial intelligence is penetrating most of the classification and recognition tasks performed by a computer. This work proposes to classify flower images based on features extracted during segmentation and after segmentation using multiple layered neural networks. The segmentation models used are watershed, wavelet, wavelet fusion model, supervised active contours based on shape, color and Local binary pattern textures and color, fused textures based active contours. Multi-dimension feature vectors are constructed from these segmented results for each indexed flower image labelled with their name. Each feature becomes input to a neuron in various feature layers and error back propagation algorithm with convex optimization structure trains these multiple feature layers. Testing with different flower images sets from multiple sources resulted in average classification accuracy of $92 \%$ for shape, color and texture supervised active contour segmented flower images.
\end{abstract}

Keywords: Flower Image Segmentation; Pattern Classification; Multi-Dimensional Features; Artificial Neural Networks.

\section{Introduction}

Image classification is a vibrant area of research in image understanding and computer vision. Abundant classifiers have been proposed in literature for different applications. In this paper, we propose to use some of these models of segmentation, feature extraction and classification on flower images. Flowers are a complete natural representation of color, texture and shape features processed under the sun. Flower images are captured with different variations in cameras, angles and lighting conditions. Segmenting for flower extraction and classifying the images is a challenging task.

In our previous works [1][2][3], we have segmented the flower images successfully using watershed (WF), marker controlled watershed (MCWF), wavelets (HWF), canny (CF), canny - watershed fusion (CWFF), active contours with shape, color, texture priors (ACSCTF) and fused color - texture featured active contours (ACTFCF). In this work, we propose to use these segmented flower images and extract features for classification using multi layered artificial neural networks. The proposed features are Zernike moments (ZM), Hu moments (HM), Haar shape features (HSF), Local Binary pattern (LBPTF) features and their combinations.

Natural images are difficult to segment using traditional image processing techniques. They contain a wide variety of colors, textures and shapes tightly binding with each other. A distinct boundary or region labelling becomes a challenging task [4] for segmentation algorithms [5]. Advanced image segmentation algorithms depend on color, shape and texture descriptors during problem assignment [6]. Natural image segmentation has long focused on texture features for dividing image into texture regions. All the methods in literature suffer in two aspects: (1) segmentation ap- proach and (2) texture feature selection. The segmentation algorithm quality is measured based on these two problems.

The texture feature alone will not represent image regions accurately in natural flower images. Color is a feature that can affect the segmentation process in along with texture [7]. Extracting color features for image region description is considered a complex problem on different color planes. Research shows different color models represent image regions with unique color information for better segmentation [8].

Natural images are rich in color and texture information with high pixel density. Segmenting regions from these images in considered a complicated task in image processing. In this work, we focus on flower image segmentation from images captured using a digital camera. The flower images captured are imperfect in accepts such as brightness, contrast, flower capturing distance, flower capturing angle, number of flowers, size of flower and background. The segmentation problem should also handle the above problems apart from extracting flower.

A set of experiments are designed to test the proposed model on benchmark flower dataset from oxford university [8]. A similar dataset on the lines of [8] is created by us to test the proposed model. There are 102 class models of flower images in [8]- [11] and 30 class models in our own dataset making a total of 132 classes. The proposed model is tested on all 132 classes of flowers to gauge the performance of the multi layered neural network classifier. We test the algorithm against 3 different layered ANN's with 3 different sets of features.

\section{Literature Review}

In [9], a standard visual vocabulary of flower species is created as images in multiple variations under a single flower name as label. The dataset is named as Oxford University flower dataset (OUFD) 
and on the similar lines we have created a flower dataset named as KL University Flower Dataset (KUFD). The OUFD consists of 102 species of flower and KUFD has 32 species of flowers respectively.

The first process in the flower classification problem focuses on image segmentation. In [10] and [11], the authors use a supervised model based flower textures with graph cuts to extract flower content. This process models textures of flower as patch models and the model guides the graph cuts algorithm to initiate the minimum cut at the texture maximum locations in the flower image. The authors in [12] use color clustering and shape features to perform region of interest (ROI) based flower image retrieval. The color clustering is achieved using color histogram based features. Shape feature set is defined based on centroid contour distance (CCD) and Angle Code Histogram (ACH) characterizing the flower contours. They have tested the algorithm on 885 flower images from 14 species.

A completely unsupervised model is proposed in [13] with simple color based thresholding. RGB color space is converted into Lab color space and OTSU thresholding is performed on all threecolor spaces. The best thresholded flower image is selected which is close to ground truth image. The authors claim that their model is faster compared to [10].

In [14], authors use a foreground background model based on Laplacian propagation algorithm computing confidence values of the pixels belonging to foreground or background. The segmentation is tested on 578 flower species with 250000 images and 102 flower species of OUFD with stable segmentation results. The results in [15] are based on the contour matching algorithm of both flower and leaf images. The results are not very encouraging. The authors in [16] uses an interactive flower segmentation model based on color and shape features. The user must draw a bounding box on the location of flower and the segmentation algorithm uses a flower boundary tracing algorithms extracts the flower regions more accurately. Experiments were conducted on OUFD and results show a near accurate boundary detection on a large set of images.

A large set of features such as color, texture and shapes of flowers is used to classify flower images in [17]. The spatial distribution of features is then classified with support vector machine (SVM) classifier. The testing is initiated on Caltech 101/256 dataset with single feature and multiple feature kernels and found an improvement from $55.1 \%$ for single feature to $72.8 \%$ for multiple features. Gray level co-occurrence matrix (GLCM) and gabor texture features are combined from a dataset 1250 flower images and classified with KNN classifier in [18]. The flower images are extracted from the internet flower image search. In [19], color and shape features are modelled as a twostep segmentation process. The model is immune to viewpoint changes and petal deformations across different flower classes. The segmentation is produced with Markova random fields (MRF) cost function optimization.

Color image segmentation is used to monitor flower growth in nature with image processing [20]. Flower images used are Lesquerella flowers for oil production. HSI color model with Monte Carlo approach is used for image segmentation. In [21] rose curve based interactive computer visual segmentation model is used effectively for flower classification.

Mobile applications are also proposed with android applications for flower classification. Mobile based flower recognition with Difference Image Entropy (DIE) and contour features of the flower from the original flower images. The average recognition is $95 \%$ with an average run time $9033 \mathrm{~ms}$ on mobile platforms [22]. In [23], Neural networks are employed on texture features to classify 1800 flower images on 30 varieties. A content based image retrieval system to characterized flower images efficiently. ANN with backpropagation is used as a classifier effectively to recognize flower species.

In this work, we propose to use a multiple segmentation models with multiple features on a ANN classifier [24] [25]. Here we use watershed (WF), marker controlled watershed (MCWF), wavelets (HWF), canny (CF), canny - watershed fusion (CWFF), active contours with shape, color, texture priors (ACSCTF) and fused color - texture featured active contours (ACTFCF). In this work, we propose to use these segmented flower images and extract features for classification using multi layered artificial neural networks. The proposed features are Zernike moments (ZM), Hu moments (HM), Haar shape features (HSF), Local Binary pattern (LBPTF) features and their combinations. ANN multi-layer backpropagation training and testing algorithm is used on 102 OUFD classes and 32 KUFD flower datasets. The algorithms are compared for accuracy and recall efficiency.

\section{Methodology}

The proposed methodology involves, flower image segmentation models, feature extractions and classifier.

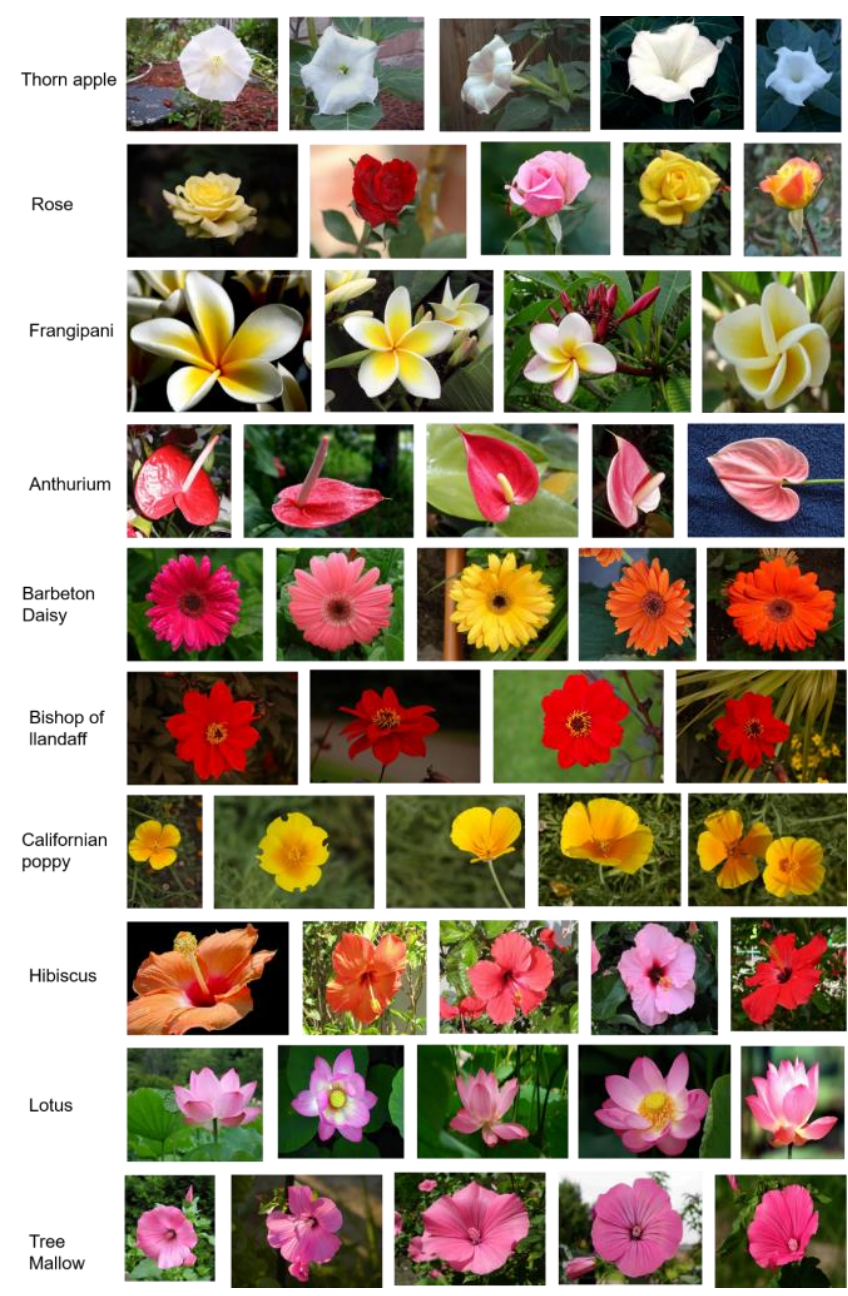

Fig. 1: Oxford university flower dataset (OUFD) flower images with class labels.

\subsection{Flower image segmentation models}

Seven segmentation algorithms based on gradient models, color, texture and shape features with active contours are proposed for flower image segmentation. The flower datasets used are shown in fig.1 and fig.2. Fig.1 shows dataset images from OUFD and fig.2. are from KUFD.

The flower image segmentation algorithms are discussed exclusively in our previous works at [1] - [3]. Here we present a brief overview of two methods based on the results obtained during segmentation. Active contour models are presented from our previous literature in this work. The latest model is based on the color, multi texture fusion model with level set formulation is discussed in this work exclusively. 


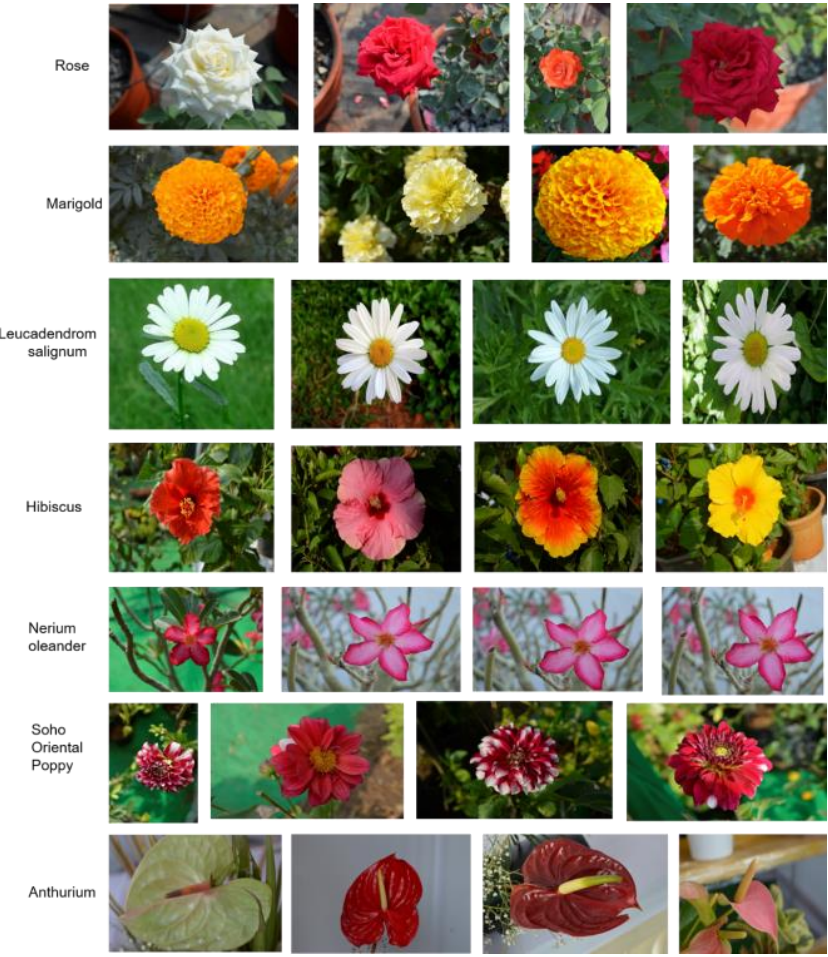

Fig. 2: KL University Flower Dataset (KUFD) with labels

\subsection{Fused color - texture featured active contours (ACTFCF)}

The flow chart for the flower segmentation process is shown in fig.3. Here we propose to use two kinds of colored texture features of the flower image representing distinct texture representations. Color covariance matrix (CLCM) represents colored textures using a probability matrix of co-occurring pixels in RGB planes [26]. It represents the random variations in pixel values to measure texture parameters in 4 directions. In color gabor texture (CGT), the image is model in frequency and orientations with Gaussian kernels. CLCM represents a texture existence and CGT represents texture regularity.

Both are necessary for flower identification in human visual system(HSV) along with flower color. Color information is embedded in RGB planes and we calculate texture features on each color plane. Apply principle component analysis max fusion on CLCM and CGT features and develop a complete color and texture representation of flower object. A global convex energy minimization framework using the fused colored texture flower object is proposed to segment flower from natural unconstrainted photos.

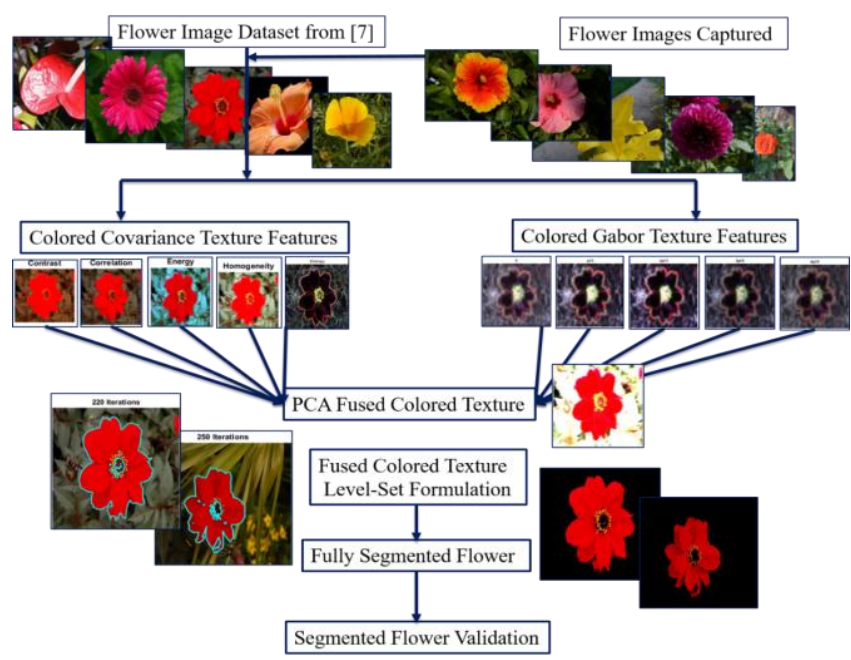

Fig. 3: Flow chart for the Fused color - texture featured active contours (ACTFCF)
More than 16 statistical parameters are defined in [27] and the list is increasing. For generating color co-occurrence probability matrix, we use the same procedure as GLCM on all RGB color planes computed as

$\operatorname{CLCM}(d, \theta, 3)=p_{r}(x, y, 3 / d, \theta, g, N)$

In this paper, we use 4 pixel distances in 4 different orientations

$$
(d, \theta)=\left[\begin{array}{l}
(\{1,2,3,4\}, 0),\left(\{1,2,3,4\}, \frac{\pi}{4}\right), \\
\left(\{1,2,3,4\}, \frac{\pi}{2}\right),\left(\{1,2,3,4\}, 3 \frac{\pi}{2}\right)
\end{array}\right] .
$$

The smaller distances capture local information and larger distances capture distinct textures. For each value of $d$ and $\theta$ we build co-occurrence matrices and use them to calculate the structural statistics. In this work, we calculated 14 parameters. However, for the flower image set chosen [7], only 5 parameters found to useful. They are: contrast, energy, entropy, homogeneity and correlation.

A 2D gabor filter [28] is a Gaussian kernel modulated with sinusoidal plane wave represented as

$$
\begin{aligned}
& G_{k}\left(x, y \mid \omega, \theta, \varphi, c_{x}, c_{y}\right)= \\
& \exp \left(\frac{-\left\{\left(x-c_{x}\right)^{2}-\left(y-c_{y}\right)^{2}\right\}}{2 \sigma^{2}}\right) \times \sin (\omega(x \cos \theta-y \sin \theta)+\varphi) .
\end{aligned}
$$

Where, $\sigma$ is Gaussian variance, $\theta$ is filter angle, $\omega$ is frequency and $\varphi$ is phase shift. $\left(c_{x}, c_{y}\right)$ gives the filter centre. For a flower image $I(x, y)$, the gabor spectrum is calculated as a linear convolution of $I \otimes G_{K}$. The spectrum detects gabor texture features in 5 different orientations in this work. Phase changes and frequency changes are fixed for flower images as there is texture consistency in these parameters. Hence only orientation changes are considered. Five different orientations are used to match that of CLCM. They are $\left\{0, \frac{\pi}{5}, 2 \frac{\pi}{5}, 3 \frac{\pi}{5}, 4 \frac{\pi}{5}\right\}$ by defining the gabor texture exactly as

$G_{K\{1, \ldots, 5\}}=\sum_{x y}^{S(x y)} G_{k}\left(x, y \mid \omega,\left\{0, \frac{\pi}{5} . ., 4 \frac{\pi}{5}\right\}, \varphi, c_{x}, c_{y}\right) \otimes I(x, y)$.

The symbol $\otimes$ is a convolution operator. The parameter $G_{K\{1, \ldots, 5\}}$ represent gabor texture features of the flower image. To make Gabor texture as a color gabor texture (CGT), we apply the convolution operator on the 3 planes of the image. The process is defined as

$$
G^{C}{ }_{K\{1, \ldots, 5\}}=\bigcup_{C=R G B}\left(\sum_{x y}^{S(x y)} G_{k}\left(x, y \mid \omega,\left\{0, \frac{\pi}{5} . ., 4 \frac{\pi}{5}\right\}, \varphi, c_{x}, c_{y}\right) \otimes I^{C}(x, y)\right) .(5)
$$

This filter configuration covers the entire spatial - frequency plane where every pixel responds to every filter.

To make a distinct texture feature a linear feature combiner is used [29]. Principle component analysis based linear combiner is espoused. The 20D CLCM and 5D CGT are PCA fused with a decision on higher frequency components of gabor components defined as

$$
\left.\begin{array}{l}
P C A_{C G T}(f)=\frac{e i g V(C G T)}{\sum \operatorname{eig} V(C G T)} \forall \operatorname{eig}(C G T(f)) \geq e i g(C L C M(f)) \\
P C A_{C L C M}(f)=\frac{e i g V(C L C M)}{\sum e i g V(C L C M)} \forall e i g(C G T(f)) \leq e i g(C L C M(f))
\end{array}\right\} .
$$

For each feature vector $f$. The linear combination of principle components for each dimension are combined using the expression 


$$
F_{c f f}=P C A_{C L C M}(f) C L C M(f)+P C A_{G C T}(f) C G T(f) .
$$

In [29], a 25 D fused texture feature is constructed to represent all the frequency components in the texture feature. In this work a linear operator in eq'n 13 combines all the $25 \mathrm{D}$ texture feature into single texture descriptor of size $S(x) \times S(y) \times 3$. This fused color texture feature is used as supervised input to a convex energy minimization active contour framework.

The colored texture invariance of the prior textures is moved by

$$
E_{c t}(\varphi)=d^{2}\left(\varphi_{0}, \varphi_{t}\right)=\iiint_{\theta}\left(-H\left(\varphi_{0}\right)+H\left(F_{c t f}\right)\right)^{2} d c
$$

where $C$ defines RGB color planes. The curve evolution expression is obtained by applying Euler Lagrange eq'n, the colored texture level set evolution expression is

$$
\begin{aligned}
\frac{\partial \varphi}{\partial t}=\tau_{c} \delta(\varphi)\left[\lambda\left(\nabla \cdot \frac{\nabla \varphi}{|\nabla \varphi|}\right)\right. & \left.-\left(F-\varphi_{i}\right)^{2}+\left(F-\varphi_{e}\right)^{2}\right] \\
& +\left(1-\tau_{c}\right)\left(-H\left(\varphi_{0}\right)+H\left(F_{c t f}\right)\right)^{2} .
\end{aligned}
$$

The above equation is simulated first on the synthetic texture images and then a set of experiments are designed using benchmark flower datasets and our dataset using the above colored texture fused driven level set segmentation model. The delta function is $\delta(\theta)$ and eq'n 9 is updated iteratively as

$$
\varphi_{i}=\frac{\iint_{\theta} F H(\theta) d s}{\iint_{\theta} H(\theta) d s} \text { and } \varphi_{e}=\frac{\iint_{\theta} F(1-H(\theta)) d s}{\iint_{\theta}(1-H(\theta)) d s} .
$$

The other flower segmentation models are inherited from [1], [2] and [3].

\section{Multi Feature Extraction - Zernike, $\mathrm{Hu}$ Moments, LBP, Haar}

A set of 4 features are used to represent the flower characteristics in the segmented images. They are Zernike moments (ZM), Hu moments (HM), Haar shape features (HSF), Local Binary pattern (LBPTF) features and their fusion.

\subsection{Zernike Moments}

ZM [30] of order $n$ with repetition $m$ for continuous feature set $F_{s}^{t}(x, y)$ per frame $t$ over a unit disk is defined as

$$
M_{n m}=\frac{n+1}{\pi} \int_{\text {Unit Disk }} Z_{n m}^{*}(x, y) F_{s}^{t}(x, y) d x d y
$$

If $\theta_{0}$ is the rotational angle, with original $\mathrm{ZM}$ and rotated $\mathrm{ZM}$ as $A_{n m}$ and $A_{n m}^{R}$, respectively. We have

$$
\left|M_{n m}^{R}\right|=\left|M_{n m} e^{-j m \theta_{0}}\right|=M_{n m} ; \quad \Theta_{n m}^{R}=\Theta_{n m}-m \theta_{0}
$$

Where $\left|M_{n m}\right|$ and $\Theta_{n m}$ represent magnitude and phase, respectively. In (12) the magnitude remains constant while the image rotates, whereas the phase changes with image rotation. Hence, most of the applications use ZM magnitude as a feature vector for pattern classification. In this work, we propose to ZM magnitude on 2D shape point cloud as invariant feature representing the small variations in camera movement that occur during flower image capture in natural environments. To represent changes in the flower image dimensions which happen non-linearly, we propose to use a nonlinear function defined over geometric moments.

\subsection{Hu Moments}

$\mathrm{Hu}$ moments in [31] are non - orthogonal centralized moments that are scale, translation and rotation invariant. Human dancers come in all shapes and sizes and the features describing them may change with dancer. Modelling invariance in dancer shape features from the 2D point segments of fig.5(e) or (j) is done with $\mathrm{Hu}$ moments. Hu moments are derived for 2D normalized central moments $M_{p q}^{H}$ using algebraic invariants:

$$
\begin{aligned}
\hbar_{1}= & M_{2,0}+M_{0,2} \\
\hbar_{2}= & M_{2,0}-M_{0,2}-4 M_{1,1}^{2} \\
\hbar_{3}= & \left(M_{3,0}-3 M_{1,2}\right)^{2}+\left(3 M_{2,1}-M_{3,0}\right)^{2} \\
\hbar_{4}= & \left(M_{3,0}+M_{1,2}\right)^{2}+\left(M_{2,1}+M_{0,3}\right) \\
\hbar_{5}= & \left(M_{3,0}-3 M_{1,2}\right)\left(M_{3,0}+M_{1,2}\right)\left[\left(M_{1,2}+M_{3,0}\right)^{2}-\left(M_{2,1}+M_{0,3}\right)^{2}\right] \\
+ & \left(3 M_{2,1}-M_{3,0}\right)\left(M_{2,1}+M_{0,3}\right)\left[3\left(\left(M_{3,0}+M_{1,2}\right)^{2}-\left(M_{3,0}+M_{1,2}^{2}\right)\right)\right] \\
\hbar_{6}= & \left(M_{2,0}-M_{0,2}\right)\left[\left(M_{3,0}+M_{1,2}\right)^{2}-\left(M_{2,1}+M_{0,3}^{2}\right)\right] \\
& \quad+4 M_{11}+\left(M_{3,0}+M_{1,2}\right)\left(M_{0,3}+M_{2,1}\right) \\
\hbar_{7}= & \left(3 M_{2,1}-M_{3,3}\right)\left(M_{3,0}+M_{1,2}\right)\left[\left(M_{1,2}+M_{3,0}\right)^{2}-\left(M_{2,1}+M_{0,3}\right)^{2}\right] \\
& \quad-\left(M_{3,0}-3 M_{1,2}\right)\left(M_{2,1}+M_{0,3}\right)\left[3\left(\left(M_{3,0}+M_{1,2}^{2}\right)-\left(M_{2,1}+M_{0,3}\right)^{2}\right)\right]
\end{aligned}
$$

These 7 moments are calculated for every hand shape in the frame.

\subsection{Local Binary Pattern Features}

LBP compares each pixel in a pre-defined neighbourhood to summarize the local structure of the image. For an image pixel $F^{t}(x, y) \in \mathfrak{R}^{+}$, where $(x, y)$ gives the pixel position in the intensity image. The neighbourhoods of a pixel can vary from 3 pixels with radius $r=1$ or a neighbourhood of 12 pixels with $r=2.5$. The value of pixels using LBP code for a centre pixel $\left(x_{c}, y_{c}\right)$ is given by

$$
\begin{aligned}
& L_{S}^{t}=L B P\left(x_{c}, y_{c}\right)=\sum_{j=1}^{P} F^{t}\left(g_{p}-g_{c}\right) 2^{p} \\
& F^{t}(x)= \begin{cases}1 & \forall x \geq 0 \\
0 & \text { Otherwse }\end{cases}
\end{aligned}
$$

Where $g_{c}$ is binary value of centre pixel at $\left(x_{c}, y_{c}\right)$ and $g_{p}$ is binary value around the neighbourhood of $g_{c}$. The value of $P$ gives the number pixels in the neighbourhood of $g_{c}$. The local shape descriptor $L_{S}^{t}$ of the segmented flower projects maximum number of points on to feature space.

\subsection{Haar Wavelet Features - Global Shape Descriptor}

The objective at this stage is to represent flower shape with a set of wavelet coefficients. Here we propose to use Haar wavelet at level 1. At level 1, Haar wavelet decomposes the video frame $V^{t}$ into 4 sub-bands. Fig.4. shows the 4 sub-bands at 2 levels. At $1^{\text {st }}$ level we have 4 sub-bands and at $2^{\text {nd }}$ level have 8 sub-bands. In the $1^{\text {st }}$ level, the three sub-bands represent the shape information at three different orientations: Vertical $v$, Horizontal $h$ and Diagonal $d$. Combining the three sub-bands and averaging the wavelet coefficients normalizes the large values.

$W_{S}^{t}=\frac{h+v+d}{3}$ 
The averaged shape harr wavelet coefficients $W_{S}^{t}$, along with $\{h, v, d\}$ sub-band coefficients are reconstructed to spatial domain. Fig.4. shows the reconstructed spatial domain frame producing the exact flower shapes features.

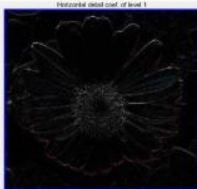

(a)

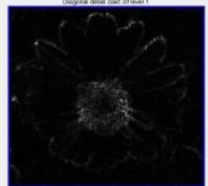

(b)

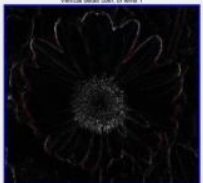

(c)

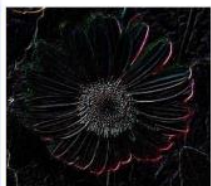

(d)
Fig. 4: Showing Haar shape coefficients. (a) Horizontal Haar, (b) Diagonal Haar, (c) Vertical Haar and (d) Averaged Haar Shape features.

\subsection{Feature Vector Representation for Multi-layer ANN}

Artificial neural network classifier is proposed for recognizing flower classes from a set of pre-rained example classes. The Zernike and $\mathrm{Hu}$ moments form a feature vector, where each neuron is inputted with each moment. However, for LBP and Haar features appear in the form of a matrix. These 2D features are reshaped to form a 1D features. By trial and error, the number of features for flower characterization using LBP is 112 values from LBP matrix and 72 values for Haar matrix. ZM is calculated on each segmented flower in 4 directions resulting in 4 magnitudes and 4 orientations. For $\mathrm{Hu}$ moments, a standard set of 7 features are extracted by using equations in (13). A total of 199 feature values are used to represent a flower image.

\section{ANN Classifier}

The details of ANN with back propagation algorithm are listed in our previous work at [32] and the models used for coding are considered from [33] and [34]. Further we also study the usefulness of multilayer feed forward ANN's with more than 5 layers along with their performance analysis on sign classifications. A system that can handle large data matrices is the feed-forward ANN [31].

The dimensionally reduced feature matrix $f^{v}$ serves as the input for training the feed-forward NN, as shown in Fig.5.

A typical feed-forward $\mathrm{NN}$ is a combination of three layers of neurons: input layer, hidden layer, and output layer. The neurons in these layers are activated using a nonlinear sigmoid activation function [34-37]. Let $x_{i, j}(i t r)$, where $1 \leq i \leq N, 1 \leq j \leq M$, be the input to the $\mathrm{NN}$ derived from feature matrix $f^{v}$. M and $\mathrm{N}$ denote the number of columns and rows of $f^{v}$, and itr is the number of iterations, also called epochs. ${ }^{y_{i, j}}($ itr $)$ denotes the NN outputs, where $1 \leq i \leq N, 1 \leq j \leq M$

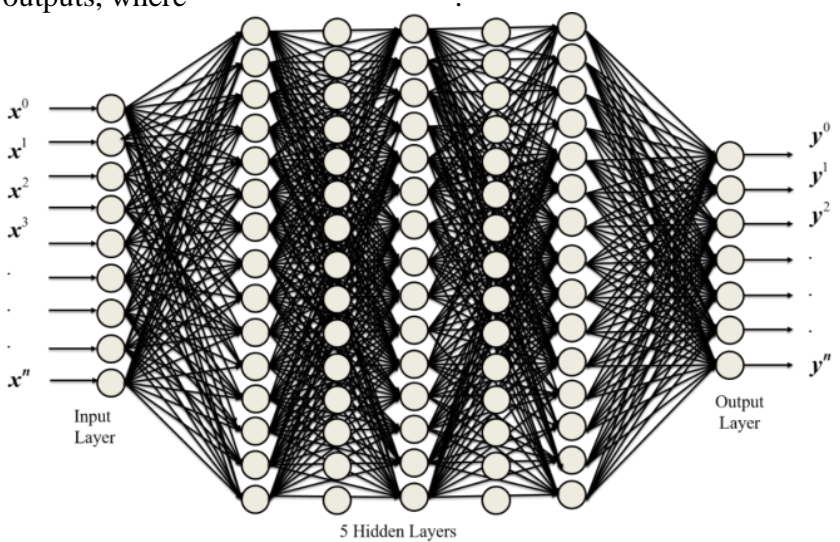

Fig. 5: A 5 layer ANN

An ANN was employed for recognizing and classifying flower images. First, the NN was trained with 102 flower image classes represented with 199 features. Consequently, the network had 199 neurons in the input layer and 102 neurons in the output layers in addition to 156 neurons in the hidden layers. This NN object can handle 102 input images and classify them into 102 signs.

\section{Experimentation and Results}

Two experiments were designed to test the robustness of the proposed segmentation models and the corresponding extracted features. The first experiment answers the question related to which flower segmentation algorithm performs well with the proposed ANN classifier. The second experiment focus on using a single feature or bag of features at the input of the classifier for flower classification. We use three performance evaluators for validating the results. They are accuracy - recall curves and accuracy rate per flower class. For a strong hypothesis $H$ resulted from ANN training and testing for an input feature $f_{i}$ on a trained distribution $D$ with $Z_{i}=H\left(f_{i}\right)$ predicted labels. The following metrics in [38-40] are

$$
\begin{aligned}
& \operatorname{accuracy}(H, D)=\frac{1}{|D|} \sum_{i=1}^{|D|} \frac{\left|L_{i} \cap Z_{i}\right|}{\left|Z_{i}\right|} \\
& \operatorname{Recall}(H, D)=\frac{1}{|D|} \sum_{i=1}^{|D|} \frac{\left|L_{i} \cap Z_{i}\right|}{\left|L_{i}\right|}
\end{aligned}
$$

\subsection{Experiment - 1: For segmentation models}

In exp-1, we use 7 segmentation models for the flower dataset in [7]. They are watershed (WF), marker controlled watershed (MCWF), wavelets (HWF), canny (CF), canny - watershed fusion (CWFF) [1],[2] , active contours with shape, color, texture priors (ACSCTF) [3] and fused color - texture featured active contours (ACTFCF) is proposed in this work.

The flower dataset consists of 102 classes with multiple instances of those classes. Each flower class has multiple flower images varying from numbers 10 to 20 . For training the ANN we have initiated 5 flower images per class. The training vectors change from feature to feature. Table 1 gives the size of training vectors generated from a single feature vector.

Table 1: ANN training matrix size from features used in this work

\begin{tabular}{c|c}
\hline Feature Vector Algorithm & $\begin{array}{c}\text { ANN Training Vector - Indicating } \\
\text { Number of Columns }\end{array}$ \\
\hline ZM & $102 \times 8 \times 5$ \\
HM & $102 \times 7 \times 5$ \\
LBPTF & $102 \times 112 \times 5$ \\
HSF & $102 \times 72 \times 5$ \\
All 4 Features & $102 \times 199 \times 5$ \\
\hline
\end{tabular}

The reaming flowers in the class models are used for testing. The target matrix is a binary labelled matrix which is characterized by flower class names. The segmentation results from active contours with shape, color, texture priors (ACSCTF) [3] and fused color texture featured active contours (ACTFCF) are shown in figures 6 and 7 respectively. 


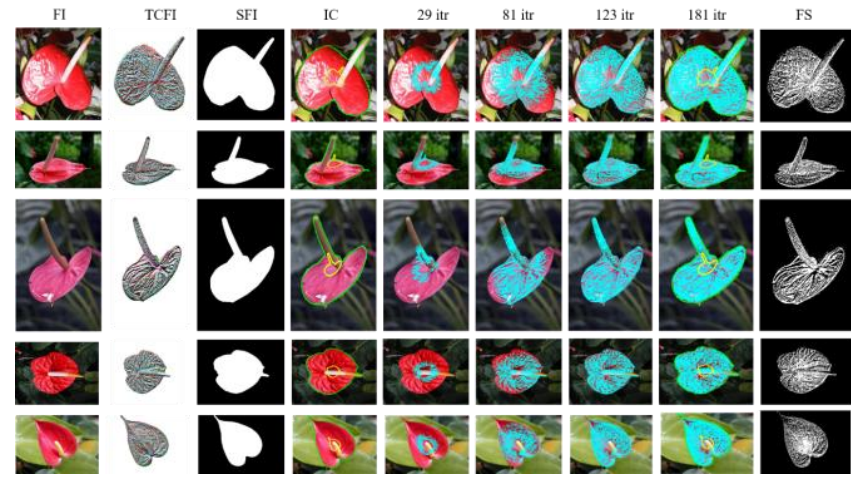

Fig. 6: Results of active contours with shape, color, texture priors (ACSCTF) [3]

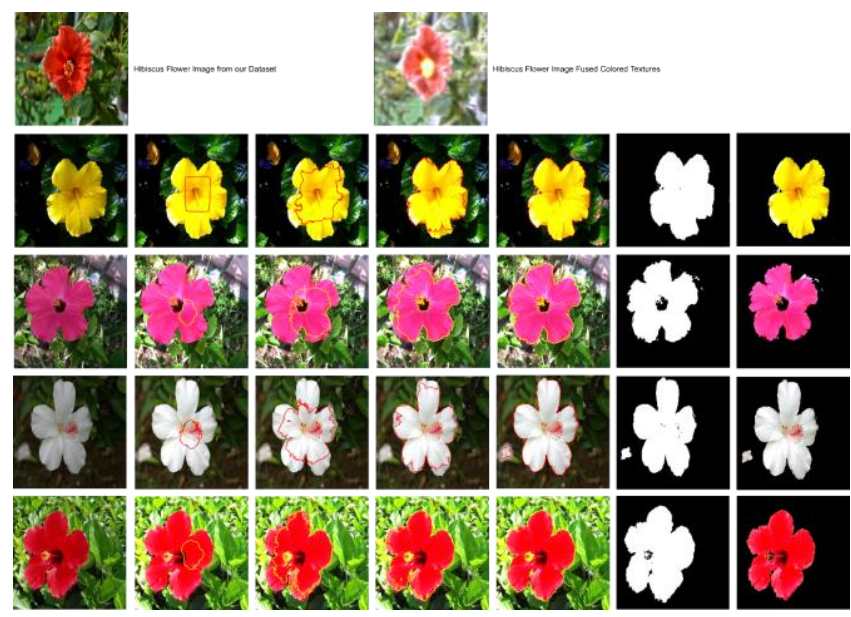

Fig. 7: Showing results with Fused color - texture featured active contours (ACTFCF)

Features extracted from the segmentation outputs from 7 algorithms of 102 flower images are fed into the input layer of ANN in fig.5. Each network is trained separately with a specific feature vector. Back propagation algorithm is used for training all ANN modules. To maintain commonality between the comparisons the ANN 5-layer model is used for all types of segmentations and features.

The training process requires a set of examples of appropriate network behaviour, network inputs, and target outputs. During training, the weights and biases of the network were iteratively adjusted to minimize the network performance function, the mean squared error (MSE) in the case of feed-forward networks.

The network was trained with $102 \times 5$ samples for 102 flower class images under different conditions for a segmentation algorithm with 4 features separately. A total of 8695 epochs were exercised for training for the segmentation algorithm discussed in this work with mixed features. The system was tested with 5 flower images that were previously unseen by the network in the testing phase. During the testing phase, the network was tested more than once. The MSE tolerance was fixed at 0.0001 for sample training. The learning rate and momentum factor were selected as 0.25 and 0.9 , respectively. The hidden and output neurons were activated using a hyperbolic tangential sigmoid transfer function. A graph of the MSE against the epoch was plotted in fig.8.

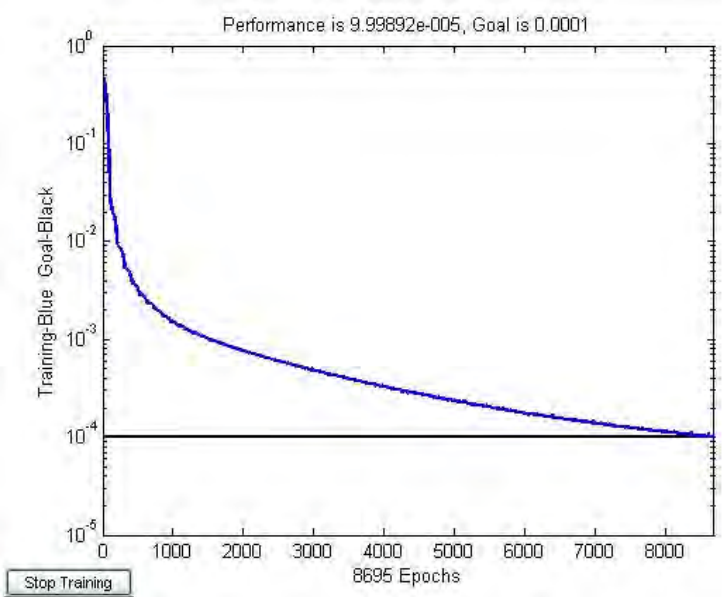

Fig. 8: Graph of mean squared error versus epoch for $102 \times 5$ flower training classes

Table 2. Gives the number of epochs for each segmentation with different features.

\begin{tabular}{cccccc}
\hline \multirow{2}{*}{$\begin{array}{c}\text { Segmentation } \\
\text { Algorithm }\end{array}$} & \multicolumn{5}{c}{$\begin{array}{c}\text { Number of Epochs for training used by } 5 \text { hidden layer } \\
\text { network with different features }\end{array}$} \\
\cline { 2 - 6 } & ZM & HM & LBPTF & HSF & $\begin{array}{c}\text { All } 4 \\
\text { Features }\end{array}$ \\
\hline WF & 1122 & 1011 & 4523 & 3562 & 6552 \\
MCWF & 1322 & 1081 & 4623 & 3812 & 6525 \\
HWF & 1001 & 952 & 4128 & 3102 & 6212 \\
CF & 522 & 411 & 1823 & 1262 & 2152 \\
CWFF & 1422 & 1221 & 4895 & 3698 & 6854 \\
ACSCTF & 2592 & 2091 & 5523 & 4562 & 7152 \\
ACTFCF & 2812 & 2198 & 5578 & 5172 & 8695 \\
\hline
\end{tabular}

The more the training vector the better is the recognition and recall. During testing a different set of images are from a class are segmented, feature extracted and are given as input to the trained network. Testing results of each feature with proposed segmentations on separately feature trained ANN's is represented using accuracy curves. Accuracy gives the performance of the classifier is correctly recognizing the flower class. Fig.9 shows the classification accuracies computed after each testing of the ANN's.

Segmentation algorithms ACSCTF and ACTFCF with preinformation such as color, texture and shape has shown high levels of accuracy with all sets of features. On the features size, LBPTF features, which preserve the local information have provided better recognition accuracies with HSF coming second. For the ACSCTF, active contour model with color, shape and texture features the recognition accuracies for all classes of flowers is around 0.92. The texture fusion segmentation algorithms also produced good accuracies averaged around 0.9. Remaining segmentation models have mixed results with recognition accuracies around 0.6. 
(a)

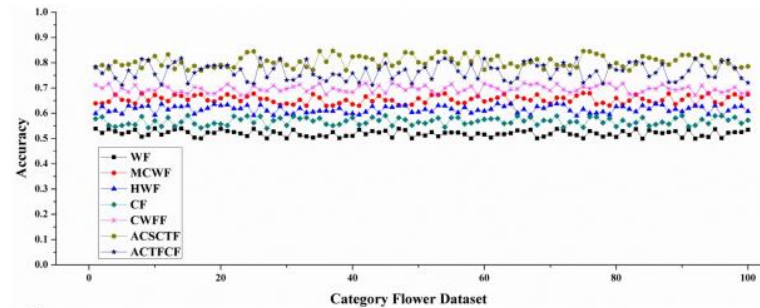

(b)

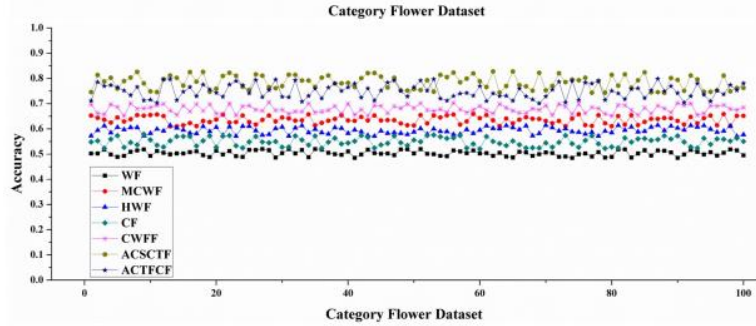

(c)

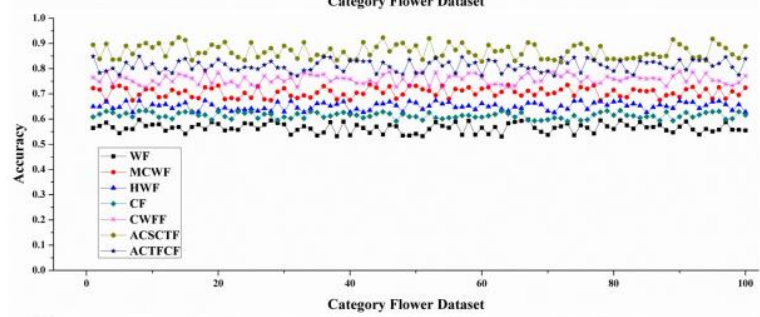

(d)

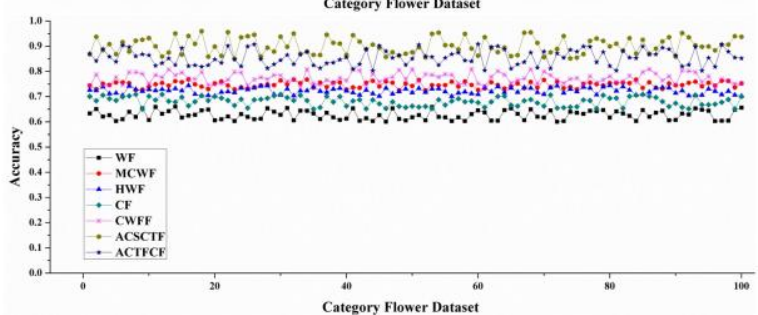

Fig. 9: Accuracy of segmentation algorithms with single feature vector trained ANN, (a) ZM, (b) HM, (c) HSF and (d) LBPTF

Segmentation algorithms ACSCTF and ACTFCF with preinformation such as color, texture and shape has shown high levels of accuracy with all sets of features. On the features size, LBPTF features, which preserve the local information have provided better recognition accuracies with HSF coming second. For the ACSCTF, active contour model with color, shape and texture features the recognition accuracies for all classes of flowers is around 0.92 . The texture fusion segmentation algorithms also produced good accuracies averaged around 0.9. Remaining segmentation models have mixed results with recognition accuracies around 0.6.

The accuracy recall curves for each of the features is calculated to investigate the recollection abilities of the ANN for different flower segmentations and features. Fig.10 shows the accuracy recall curves for each of the features used.

Segmentation algorithms ACSCTF and ACTFCF with preinformation such as color, texture and shape are showing high values on accuracy - recall plots for all features. For all other segmentation models the values are quite average and the failure rate is around $65 \%$ in case of Canny segmentation on flower images.

As the current research shows that multiple features can produce better accuracies compared to single feature models, we tested an all mixed feature model in the second experiment. This exp-2 gives exactly which segmentation model is better for segmenting flowers in natural images. The 199-feature vector is built with all 4 features per segmented flower across 102 classes in the oxford flower dataset in [7]. Fig.11 shows the accuracy plots of all 7 segmentation algorithms with mixed features. The ANN's designed for this task took more epochs for training as given in table-2.
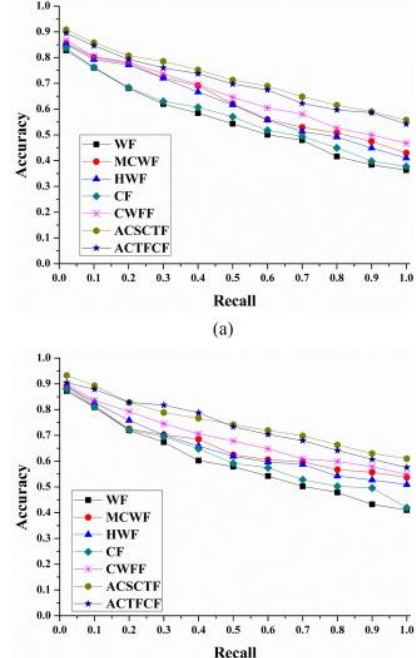

(c)

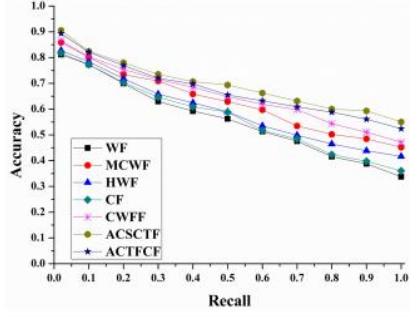

(b)

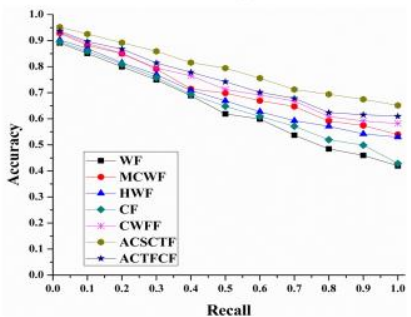

(d)
Fig. 10: Accuracy - Recall plots for 7 different segmentations with features (a) ZM, (b) HM, (c) HSF and (d) LBPTF

Compared to plots in fig.9, fig.10 shows an improvement is recognition by the ANN's. This is due to different characteristics of the flower image being represented by extracted features. ACSCTF and ACTFCF segmentation models are very accurate is recognizing over 102 flower classes compared to other models. ACSCTF recognition accuracy is averaged around 0.95 for most of the classes. ACTFCF segmentation model showed accuracy averaged around 0.9. Canny - watershed fused with wavelet CWFF is next in the line with an average accuracy of 0.85 . MCMF, marker controlled watershed segmentation is around 0.81 . The most unreliable segmentation model was WF, watershed transform at 0.7 .

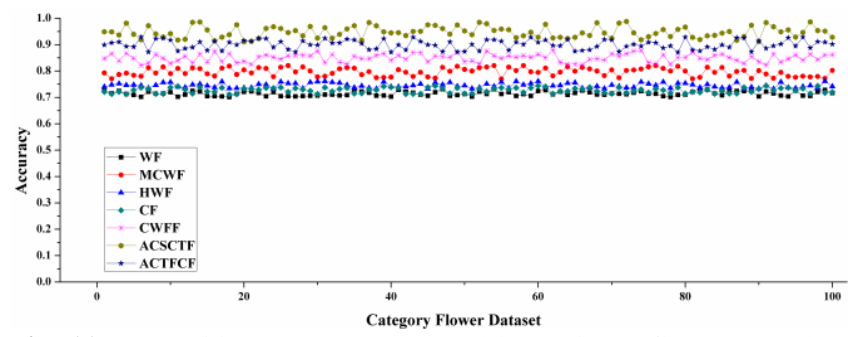

Fig. 11: Recognition accuracy computed by testing 5 flowers per class with ANN

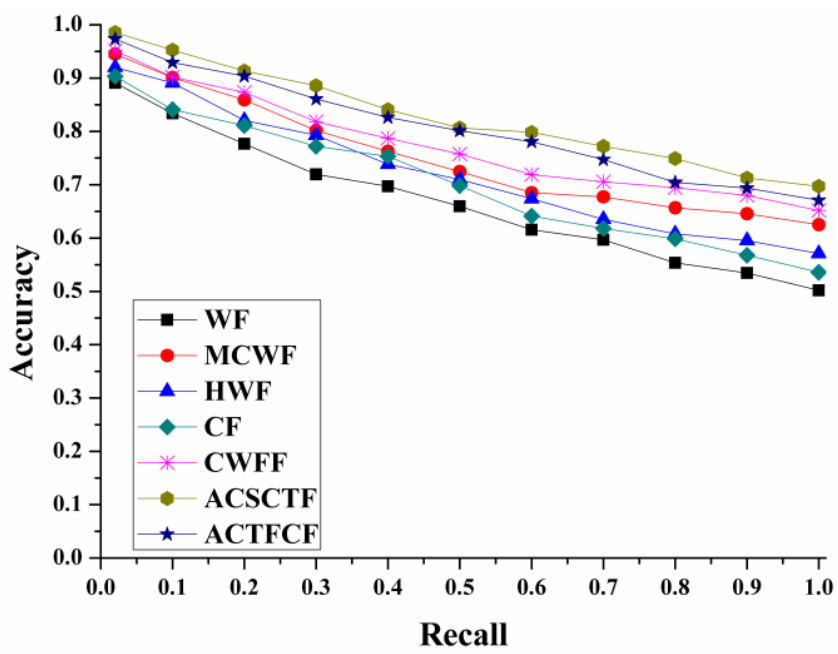

Fig. 12: Mixed feature accuracy - recall curves 
To validate the results in exp-2, accuracy - recall curves are plotted in fig. 12 for the following 7 segmentation models. The plots show ACSCTF and ACTFCF flower segmentation models perform better compared to other 5 segmentation algorithms. The algorithms almost produced $100 \%$ recall on most of the flower classes. The failure rate in multi feature model is around $5 \%$ for the two best segmentation algorithms. For other models the average failure rate is around $25-30 \%$.

We observed that, supervised segmentation algorithms performed better compared to unsupervised algorithms in segmentation of natural flower images. Similarly, multi feature combinations produced more accurate recognitions compared to single feature models. However, we obtained similar outcomes on KLU flower datasets and hence the results are same with a tolerance of $0.01 \%$ per flower class.

\section{Conclusion}

In this work, 7 flower image segmentation algorithms with 4 different features are classified with back propagation trained ANN. They are watershed (WF), marker-controlled watershed (MCWF), wavelets (HWF), canny (CF), canny - watershed fusion (CWFF), active contours with shape, color, texture priors (ACSCTF) and fused color - texture featured active contours (ACTFCF) is proposed in this work. Four (ZM), Hu moments (HM), Haar shape features (HSF) and local binary pattern texture features (LBPTF) are used on the segmented flowers. ANN is trained with single features and multiple features for each of the segmented flower classes. ANN classifier is recorded with recognition accuracy and accuracy - recall measures. The proposed method in tested on benchmark oxford flower dataset and our dataset. The active contours with shape, color, texture priors (ACSCTF) segmentation algorithm with combined features can solve complex recognition problems related to flower classification in natural unconstrained images. The proposed fused colored texture features algorithm (ACTFCF) gives satisfactory results on a wide class of flower images compared to single gray texture features.

In this section you should present the conclusion of the paper. Conclusions must focus on the novelty and exceptional results you acquired. Allow a sufficient space in the article for conclusions. Do not repeat the contents of Introduction or the Abstract. Focus on the essential things of your article.

\section{References}

[1] Inthiyaz, Syed, B. T. P. Madhav, and P. V. V. Kishore. "Flower segmentation with level sets evolution controlled by colour, texture and shape features." Cogent Engineering, vol.4, no. 1,pp.115, (2017): 1323572.

[2] Syed Inthiyaz, B. T. P Madhav, P. V. V. Kishore, Vamsi Krishna M., Sri Sai Ram Kumar M., Srikanth K. and Arun Teja B. "FLOWER IMAGE SEGMENTATION: A COMPARISON BETWEEN WATERSHED, MARKER CONTROLLED WATERSHED, AND WATERSHED EDGE WAVELET FUSION." ARPN Journal of Engineering and Applied Sciences, vol.11, no.15, pp.9382-9387, (2016).

[3] Syed Inthiyaz, B. T. P Madhav, P. V. V. Kishore, "Pre-Informed Level Set for Flower Image Segmentation", International Conference on Smart Computing and Informatics (SCI), March 2017

[4] Pont-Tuset, Jordi, Pablo Arbelaez, Jonathan T. Barron, Ferran Marques, and Jitendra Malik. "Multiscale combinatorial grouping for image segmentation and object proposal generation." IEEE transactions on pattern analysis and machine intelligence 39, no. 1 (2017): 128-140

[5] Zhang, Hui, Jason E. Fritts, and Sally A. Goldman. "Image segmentation evaluation: A survey of unsupervised methods." computer vision and image understanding 110, no. 2 (2008): 260-280

[6] Ilea, Dana E., and Paul F. Whelan. "Image segmentation based on the integration of colour-texture descriptors - A review." Pattern Recognition 44, no. 10 (2011): 2479-2501.

[7] Li, Ying, Wei Rao, Jing Peng, Ying Du, Linzhi Meng, and Zheng Gu. "Egress Mechanism Color Image Segmentation
Based on Region and Feature Fusion in Mars Exploration." In 3rd International Symposium of Space Optical Instruments and Applications, pp. 301-308. Springer, Cham, 2017.

[8] Wu, Jian Kang, Mohan S. Kankanhalli, Joo-Hwee Lim, and Dezhong Hong. "Color Feature Extraction." Perspectives on Content-Based Multimedia Systems (2000): 49-67.

[9] Nilsback, M-E., and Andrew Zisserman. "A visual vocabulary for flower classification." In Computer Vision and Pattern Recognition, 2006 IEEE Computer Society Conference on, vol. 2 , pp. 1447-1454. IEEE, 2006.

[10] Zhou, Hailing, Jianmin Zheng, and Lei Wei. "Texture aware image segmentation using graph cuts and active contours." Pattern Recognition 46, no. 6 (2013): 1719-1733.

[11] Nilsback, Maria-Elena, and Andrew Zisserman. "Delving deeper into the whorl of flower segmentation." Image and Vision Computing 28, no. 6 (2010): 1049-1062.

[12] Hong, An-xiang, Gang Chen, Jun-li Li, Zhe-ru Chi, and Dan Zhang. "A flower image retrieval method based on ROI feature." Journal of Zhejiang University-Science A 5, no. 7 (2004): 764 772 .

[13] Najjar, Asma, and Ezzeddine Zagrouba. "Flower image segmentation based on color analysis and a supervised evaluation." In Communications and Information Technology (ICCIT), 2012 International Conference on, pp. 397-401. IEEE, 2012.

[14] Angelova, Anelia, Shenghuo Zhu, and Yuanqing Lin. "Image segmentation for large-scale subcategory flower recognition." In Applications of Computer Vision (WACV), 2013 IEEE Workshop on, pp. 39-45. IEEE, 2013.

[15] Valliammal, N., and S. N. Geethalakshmi. "Automatic recognition system using preferential image segmentation for leaf and flower images." Computer Science \& Engineering 1, no. 4 (2011): 13 .

[16] Hsu, Tzu-Hsiang, Chang-Hsing Lee, and Ling-Hwei Chen. "An interactive flower image recognition system." Multimedia Tools and Applications 53, no. 1 (2011): 53-73.

[17] Nilsback, Maria-Elena, and Andrew Zisserman. "Automated flower classification over a large number of classes." In Computer Vision, Graphics \& Image Processing, 2008. ICVGIP'08. Sixth Indian Conference on, pp. 722-729. IEEE, 2008.

[18] Guru, D. S., Y. H. Sharath, and S. Manjunath. "Texture features and KNN in classification of flower images." IJCA, Special Issue on RTIPPR (1) (2010): 21-29.

[19] Nilsback, Maria-Elena, and Andrew Zisserman. "Delving deeper into the whorl of flower segmentation." Image and Vision Computing 28, no. 6 (2010): 1049-1062.

[20] Thorp, K. R., and D. A. Dierig. "Color image segmentation approach to monitor flowering in lesquerella." Industrial crops and products 34 , no. 1 (2011): 1150-1159.

[21] Zou, Jie, and George Nagy. "Evaluation of model-based interactive flower recognition." In Pattern Recognition, 2004. ICPR 2004. Proceedings of the 17th International Conference on, vol. 2 , pp. 311-314. IEEE, 2004.

[22] Kim, Jung-Hyun, Rong-Guo Huang, Sang-Hyeon Jin, and Kwang-Seok Hong. "Mobile-based flower recognition system." In Intelligent Information Technology Application, 2009. IITA 2009. Third International Symposium on, vol. 3, pp. 580-583. IEEE, 2009.

[23] F. Siraj, M. A. Salahuddin and S. A. M. Yusof, "Digital Image Classification for Malaysian Blooming Flower," 2010 Second International Conference on Computational Intelligence, Modelling and Simulation, Bali, 2010, pp. 33-38.

[24] P. V. V. Kishore, M. V. D. Prasad, D. A. Kumar, and A. S. C. S. Sastry, "Optical Flow Hand Tracking and Active Contour Hand Shape Features for Continuous Sign Language Recognition with Artificial Neural Networks," 2016 IEEE 6th International Conference on Advanced Computing (IACC), Feb. 2016.

[25] P. V. V. Kishore, D. A. Kumar, Goutham E.N.D, and M. Manikanta, "Continuous sign language recognition from tracking and shape features using Fuzzy Inference Engine,” 2016 International Conference on Wireless Communications, Signal Processing and Networking (WiSPNET), Mar. 2016.

[26] Haralick, Robert M., and Karthikeyan Shanmugam. "Textural features for image classification." IEEE Transactions on systems, man, and cybernetics 3, no. 6 (1973): 610-621

[27] Gómez, Walter, W. C. A. Pereira, and Antonio Fernando C. Infantosi. "Analysis of co-occurrence texture statistics as a function of gray-level quantization for classifying breast ultrasound." IEEE transactions on medical imaging 31, no. 10 (2012): 18891899. 
[28] Bianconi, Francesco, and Antonio Fernández. "Evaluation of the effects of Gabor filter parameters on texture classification." Pattern Recognition 40, no. 12 (2007): 3325-3335.

[29] Wu, Qinggang, Yong Gan, Bin Lin, Qiuwen Zhang, and Huawen Chang. "An active contour model based on fused texture features for image segmentation." Neurocomputing 151 (2015): 1133 1141 .

[30] Khare, Manish, Rajneesh Kumar Srivastava, and Ashish Khare. "Object tracking using combination of daubechies complex wavelet transform and Zernike moment." Multimedia Tools and Applications 76, no. 1 (2017): 1247-1290.

[31] De Bom, Clécio R., Elisângela L. Faria, P. Marcelo, P. Marcio, Maury D. Correia, and Rodrigo Surmas. "Multiscale Matching of Micro-CT images using Pattern Recognition and Hu moments." NOTAS TÉCNICAS 4, no. 1 (2016)

[32] N. Kim, "Euclidian distance minimization of probability density functions for blind equalization", in Journal of Communications and Networks, vol. 12, no. 5, pp. 399-405, Oct. 2010.

[33] V. N. Kumar and K. V. L. Narayana, "Development of an ANNBased Pressure Transducer", in IEEE Sensors Journal, vol. 16 no. 1, pp. 53-60, Jan.1, 2016.

[34] S. K. Gharghan, R. Nordin, M. Ismail and J. A. Ali, "Accurate Wireless Sensor Localization Technique Based on Hybrid PSOANN Algorithm for Indoor and Outdoor Track Cycling" in IEEE Sensors Journal, vol. 16, no. 2, pp. 529-541, Jan.15, 2016.

[35] Kishore, P. V. V., Kishore, S. R. C., \& Prasad, M. V. D. (2013) Conglomeration of hand shapes and texture information for recognizing gestures of Indian sign language using feed forward neural networks. International Journal of engineering and Technology (IJET), 5(5), 3742-3756.

[36] G. A. Rao, P. V. V. Kishore, D. A. Kumar, and A. S. C. S Sastry, "neural network classifier for continuous sign language recognition with selfie video," Far East Journal of Electronics and Communications, vol. 17, no. 1, pp. 49-71, Mar. 2017.

[37] P. V. V. Kishore, A. S. C. S. Sastry, and A. Kartheek, "Visualverbal machine interpreter for sign language recognition under versatile video backgrounds," 2014 First International Conference on Networks \& Soft Computing (ICNSC2014), Aug. 2014.

[38] K. V. V. Kumar, P. V. V. Kishore, and D. Anil Kumar, "Indian Classical Dance Classification with Adaboost Multiclass Classifier on Multifeature Fusion," Mathematical Problems in Engineering, vol. 2017, pp. 1-18, 2017.

[39] P. V. V. Kishore, D. A. Kumar, A. S. C. S. Sastry, and E. K. Kumar, "Motionlets Matching with Adaptive Kernels for 3D Indian Sign Language Recognition," IEEE Sensors Journal, pp. 1$1,2018$.

[40] K. kumar Eepuri, P. V. V. Kishore, S. A S C S, T. K. K. Maddala, and A. kumar Dande, "Training CNNs for 3D Sign Language Recognition with color texture coded Joint Angular Displacement Maps," IEEE Signal Processing Letters, pp. 1-1, 2018. 